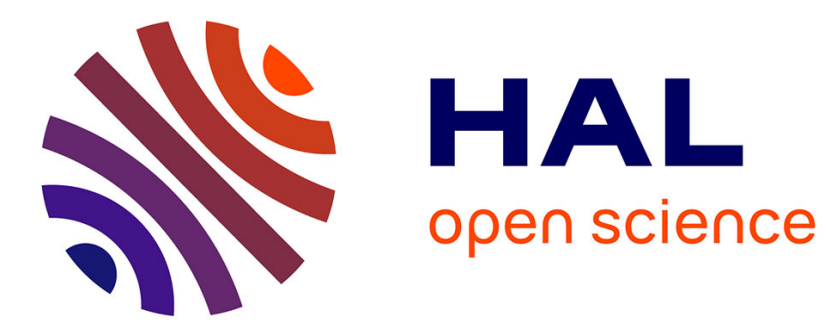

\title{
Multistability in acoustic resonance scattering
}

A. Alippi

\section{To cite this version:}

A. Alippi. Multistability in acoustic resonance scattering. Revue de Physique Appliquée, 1985, 20

(6), pp.365-368. 10.1051/rphysap:01985002006036500 . jpa-00245345

\section{HAL Id: jpa-00245345 https://hal.science/jpa-00245345}

Submitted on 1 Jan 1985

HAL is a multi-disciplinary open access archive for the deposit and dissemination of scientific research documents, whether they are published or not. The documents may come from teaching and research institutions in France or abroad, or from public or private research centers.
L'archive ouverte pluridisciplinaire HAL, est destinée au dépôt et à la diffusion de documents scientifiques de niveau recherche, publiés ou non, émanant des établissements d'enseignement et de recherche français ou étrangers, des laboratoires publics ou privés. 
Classification

Physics Abstracts

43.25

\title{
Multistability in acoustic resonance scattering
}

\author{
A. Alippi \\ Dipartimento di Energetica, University of Rome, Italy and Istituto di Acustica, CNR, Via Cassia 1216, Rome, \\ Italy
}

(Reçu le 4 octobre 1984, accepté le 8 février 1985)

\begin{abstract}
Résumé. - La diffusion du son par des structures physiques de dimensions finies à la résonance est envisagée comme un moyen possible pour l'obtention de la bistabilité dans la diffusion des ondes sonores. La dépendance non linéaire de la vitesse de propagation du son en fonction de l'intensité du son est nécessaire pour atteindre les conditions propres à la réalisation de la bistabilité. Des comportements multistables sont considérés comme pouvant survenir dans la compétition entre différentes situations physiques.
\end{abstract}

Abstract. - Resonant scattering of sound by finite physical structures is considered as a possible means of producing bistability in diffusion of sound waves. Nonlinear dependence of the sound propagation velocity upon the sound intensity is needed in order to provide proper conditions for the insurgence of bistability. Multistability behaviour is considered as a possibility in the concurrence of proper physical cases.

\section{Introduction.}

The change of the propagation velocity of a wave within a resonant structure versus the wave amplitude is a nonlinear phenomenon that may drastically affect the resonance conditions in such a way as to produce an effect of catastrophe in the structure, with resulting bistability, or multistability, behaviour. The effect has long been known in optics, where absorptive [1] and dispersive [2] effects have been alternatively investigated for the development of bistable devices. The acoustical counterpart has been experimented in surface wave propagation with hybrid structures that used non acoustical feedback mechanism [3], and it has been studied theoretically for the sake of testing proper nonlinear materials [4].

In what it follows, a generalization of the bistable resonator effect in acoustics is obtained, which takes place in scattering patterns produced by resonant structures, in case that a nonlinear change of the velocity may be assumed to take place in a strongly insonified medium. In section 2 , the resonance scattering mechanism is outlined and the fundamental results are produced, which are needed for bistability behaviour, subsequently described in section 3 , together with the qualitative conditions for the multistability behaviour.

\section{Resonance scattering.}

What is actually worth to point out in our approach to multistability is that the scattering pattern of the acoustic radiation produced by medium inhomogeneities is of the resonance type, that is, it strongly depends upon the radiation wavelength. In this respect, the simplest structure to think of - which cannot be actually considered as a scattering structure - is a slab of solid material crossed by a plane wavefront propagating in the surrounding fluid medium. The resonance conditions, in this case, cause the transparency function of the slab to reach - at some impinging angles $\vartheta$ of the incident beam to the plate normal - a maximum value, each time the wavelength to the plate thickness ratio is such that a free plate, or a Lamb wave, is excited in the plate [5]. For these conditions, the acoustic intensity reaches maximum values inside the plate and a velocity change may turn out that, through the feedback mechanism of the resonance, affects the transparency function itself, thus producing an instability effect, to be described later.

Most generally, a spatially limited object scatters an impinging wavefront away into an angular pattern, that depends on the space dimension of the object ratioed to the radiation wavelength. 
The transparency function at angular frequency $\omega$ of a plate, of thickness $d$, density $\rho$, acoustic wave velocities $c_{1}, c_{\mathrm{t}}$ for dilatational and transversal waves, respectively, submerged in a fluid medium with density $\rho_{\mathrm{f}}$ and dilatational acoustic wave velocity $c_{\mathrm{f}}$, can be written as :

$$
T=i \tau\left(\frac{1}{C_{\mathrm{s}}-i \tau}+\frac{1}{C_{\mathrm{a}}+i \tau}\right)
$$

where :

$$
\begin{gathered}
\tau=\frac{\rho_{\mathrm{f}} c_{\mathrm{f}} / \cos \vartheta}{\rho c_{\mathrm{1}} / \cos \vartheta_{1}}, \\
\left\{\begin{array}{l}
C_{\mathrm{s}} \\
C_{\mathrm{a}}
\end{array}\right\}=\cos ^{2} 2 \vartheta_{\mathrm{t}}\left\{\begin{array}{c}
\cot \delta \\
\tan \delta
\end{array}\right\}+ \\
+r^{2} \sin 2 \vartheta_{1} \sin 2 \vartheta_{\mathrm{t}}\left\{\begin{array}{c}
\cot \varepsilon \\
\tan \varepsilon
\end{array}\right\}, \\
\delta=\frac{1}{2} \times\left(n_{1}^{2}-a^{2}\right)^{1 / 2} \quad \varepsilon=\frac{1}{2} \times\left(n_{\mathrm{t}}^{2}-a^{2}\right)^{1 / 2}, \\
r=\frac{c_{\mathrm{t}}}{c_{1}} n_{1}=\frac{c_{\mathrm{f}}}{c_{1}} n_{\mathrm{t}}=\frac{c_{\mathrm{f}}}{c_{\mathrm{t}}} \\
x=k d \quad a=\sin \vartheta \quad k=\frac{\omega}{c_{\mathrm{f}}},
\end{gathered}
$$

and $\vartheta_{1, \mathrm{t}}=\sin ^{-1}\left(c_{1, \mathrm{t}} \sin \vartheta / c_{\mathrm{f}}\right)$.

Indexes s and a labelling coefficient $C$ indicate the type of wave (symmetrical, antisymmetrical, respectively), that may be excited in the plate. The structure of the transparency function $T$ is such that, for a given angle of incidence $\vartheta$ of the incoming acoustic plane wavefront, it attains a series of maxima at those reduced frequency values $x$ that make the plate to resonate. The poles of the analytical function $T$ correspond to acoustical plate, or Lamb, modes, which resonance widths depend upon the parameter $\tau$ that - apart from geometrical dependence - ratioes the acoustic impedance of the fluid to that of the solid medium. Close to a resonance peak, the transparency function can be approximated by [6] :

$$
T=\frac{\mp i \tau}{a\left(x-x_{\mathbf{m}}^{*}\right)+i \tau}
$$

where $x_{\mathrm{m}}$ are the pole locations of the free plate, and upper and lower signs refer to symmetrical and antisymmetrical modes, respectively. What is actually worth to focus is the obvious result that the resonance peak positions depend upon the speed of sound both for longitudinal and transversal waves in the plate medium. Figure 1 reports a result by Fiorito et al. [7] of function $T$ for a steel plate submerged in water, as a function of the impinging angle $\vartheta$.

Similarly, for the well known case of a cylinder submerged in a fluid medium and impinged upon by a plane wavefront parallel to the cylinder axis, a scattering function can be defined, that presents a number of poles corresponding to the acoustical

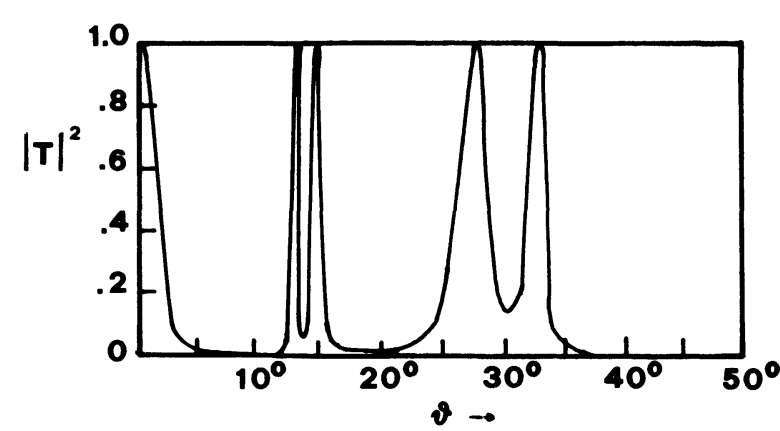

Fig. 1. - Transmission function $T$ for sound waves impinging on a steel plate from water, $v s$. the angle of incidence $\vartheta$, for $x=12$ (after Fiorito et al. [7]).

internal resonances of the wave. With the geometrical configuration given in figure 2 , the scattering function can be represented by a series of normal modes $f_{n}$ according to :

$$
p_{\text {diff }}=P_{0} \exp (-i \omega t) \sum_{0 n}^{\infty} f_{n}(x, r, \varphi),
$$

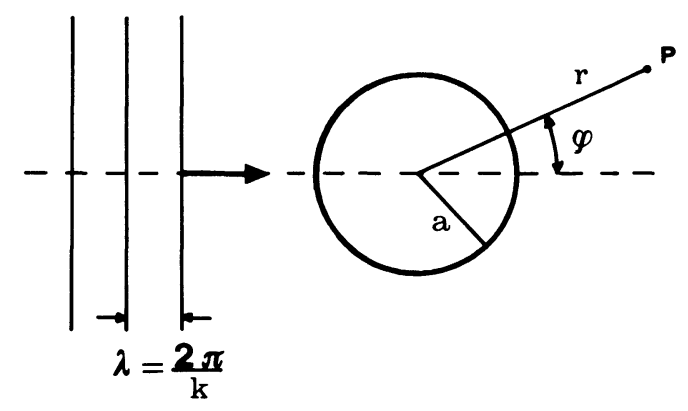

Fig. 2. - Geometry used in the formulation of the scattering problem by and infinite circular cylinder.

where the radius $a$ of the cylinder replaces the variable $d$ in the definition of $x=k a$, and $p_{\text {diff }}, P_{0}$ are pressure variables. Without going into details for the definition of functions $f_{n}$ which can be obtained from the literature [5], it only worths expressing them in terms of the transition matrix $T$, according to :

$$
f_{n}(x, r, \varphi)=\varepsilon_{n} i^{n+1} T_{n}(x) H_{n}^{(1)}(k r) \cos (n \varphi),
$$

where $\varepsilon_{n}$ is the Neumann factor $\left(\varepsilon_{n}=2\right.$ for $n=0$, $\varepsilon_{n}=1$ for $\left.n>0\right)$ and $H_{n}^{(1)}$ are the Hankle functions of the first kind. Matrix $T_{n}$ can be split into a part $T_{n}^{\mathbf{r}}$, that describes the scattering by a rigid cylinder and presents no resonances whatsoever, and a part $T_{n}^{*}$ that takes into account the resonance features of the scattering process. Each matrix $T_{n}$ presents an infinite number of poles labelled with index $l$, one for each resonance wave excited within the cylinder, and such that close to the $l$-th pole $x_{n l}^{*}$, it can be analytically approximated by

$$
T_{n}^{*}(x)=\frac{i \Gamma_{n l}}{2\left(x-x_{n l}^{*}\right)+i \Gamma_{n l}} .
$$


The poles $x_{n l}^{*}$ are the roots of equation

$$
D_{n}(x)=\left|\begin{array}{ll}
a & b \\
c & d
\end{array}\right|=0
$$

with

$$
\begin{aligned}
& a=\left(x_{\mathrm{t}}^{2}-2 n^{2}\right) J_{n}\left(x_{1}\right)+2 x_{1} J_{n}^{\prime}\left(x_{1}\right) \\
& b=2 n\left[J_{n}\left(x_{\mathrm{t}}\right)-x_{\mathrm{t}} J_{n}^{\prime}\left(x_{\mathrm{t}}\right)\right] \\
& c=2 n\left[J_{n}\left(x_{1}\right)-x_{l} J_{n}^{\prime}\left(x_{1}\right)\right] \\
& d=\left(x_{\mathrm{t}}^{2}-2 n^{2}\right) J_{n}\left(x_{\mathrm{t}}\right)+2 x_{\mathrm{t}} J_{n}^{\prime}\left(x_{\mathrm{t}}\right) \\
& x_{1, \mathrm{t}}=k_{1, \mathrm{t}} a=\frac{\omega a}{c_{1, \mathrm{t}}},
\end{aligned}
$$

and $J_{n}$ are Bessel functions of the first kind.

The resonance halfwidths $\Gamma_{n l}$ are given by :

$$
\Gamma_{n l}=\frac{2 \operatorname{Im}\left\{v_{l}\left(x_{n l}^{*}\right)\right\}}{\left.\frac{\mathrm{d}}{\mathrm{d} x} \operatorname{Re}\left\{v_{l}(x)\right\}\right|_{x=x_{n l}^{*}}},
$$

where $v_{l}$ are the roots of the equation $D_{n}=0$, with $D_{n}$ the analytical extension of function $D_{n}(x)$.

Equation (10) is then similar to the form of equation (7), where the pole positions of resonances depend upon the acoustic wave velocities within the cylinder. Examples of resonance scatterings from solid cylinders are usually presented under two forms : either the angular spectrum is plotted for a given frequency value $x$ or the dependence of function $T$ vs. $x$ is plotted for a given angular direction in space. The latter presentation is much more valuable and it is given in figure 3 for the case of retrodiffusion by an aluminium cylinder submerged in water. Resonance peaks are identified with a pair of numbers, the first one giving the value $n$, the second one $l$.

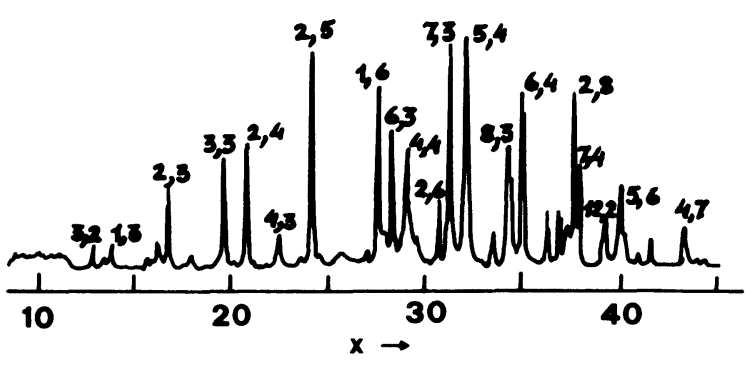

Fig. 3. - Backscattering $(\varphi=\pi)$ of sound waves by an aluminum cylinder in water. Resonance peaks are labelled with a pair of numbers, identifying variables $n$ and $l$, respectively (after Maze et al. [8]).

In complete analogy with the cylindrical case, the scattering by spherical objects can be treated, and the results formally coincide with those of equation (9), where slight differences for the parameter definitions should be allowed for [5].

\section{Scattering instabilities.}

The resonance frequency conditions outlined in the previous section are definitely specified by the value of the sound wave velocity in the scattering structure, which is in turn determined by the elastic properties of the medium. Therefore, if a dependence of the sound wave velocity exists on the sound wave amplitude, the resonance frequencies slightly shift toward higher or lower values, according to whether the sound velocity increases or decreases, respectively. An alternative interpretation is that, away from the resonance, a proper variation of the sound intensity may run the system into resonance conditions, and vice versa.

The conditions can, therefore, be achieved for the insurgence of instability effects $[1,2]$, since a positive feedback mechanism can be properly set up that accumulates energy inside of the structure following an initial increase of the sound intensity. Thereafter, the system may hang up to the resonance condition, even for a drop of the impinging sound wave intensity, before switching down to a low level state, thus behaving as a bistable system or, more generally, as a multistable one.

The dependence of the sound wave velocity upon the sound wave intensity is due to the third order elastic coefficients of the propagation medium, as it can be easily seen through the equation of the motion :

$$
\rho \frac{\partial^{2} u_{i}}{\partial t^{2}}=\frac{\partial \sigma_{i j}}{\partial x_{j}},
$$

where $\rho$ is the density of the medium, $\mathbf{u}$ is the displacement vector and $\sigma_{i j}$ the stress tensor. The constitutive equations of the medium bound stress and strain tensor

$$
u_{i j}=\frac{1}{2}\left(\frac{\partial u_{i}}{\partial x_{j}}+\frac{\partial u_{j}}{\partial x_{i}}+\frac{\partial u_{k}}{\partial x_{i}} \frac{\partial u_{k}}{\partial x_{j}}\right),
$$

through the elastic coefficients $c$ of first and higher orders :

$$
\sigma_{i j}=c_{i j k l} u_{k l}+c_{i j k l m n} u_{k l} u_{m n}+c_{i j k l m n p q} u_{k l} u_{m n} u_{p q},
$$

thus resulting into the nonlinear wave equation :

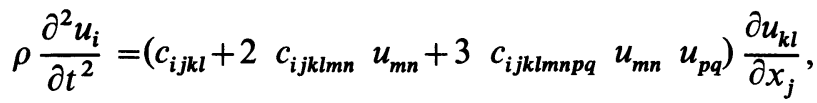

where the first term inside of the parenthesis is responsible for the linear wave velocity and the sound wave velocity can be thought to be affected by the sound wave intensity, through the additional terms. The second one is responsible for the generation of harmonic waves, and the third one for the change of sound velocity. Proper conditions for bistability effect are, then, at hand in a nonlinear medium, since the 


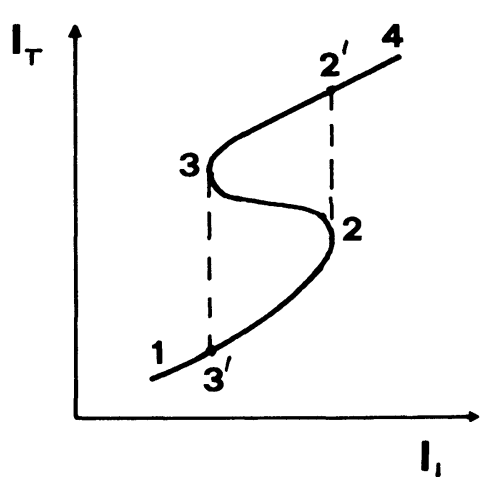

Fig. 4. - Qualitative analytical dependence of function $I_{\mathrm{T}}=I_{\mathrm{T}}\left(I_{\mathrm{I}}\right)$ in case of nonlinear scattering system : bistable behaviour produced by one resonance scattering pole.

very same transmission function $T$ depends upon the impinging sound intensity, so that the functional dependence of the transmitted, or scattered intensity $I_{\mathrm{T}}$ on the impinging one, $I_{\mathrm{I}}$ :

$$
I_{\mathrm{T}}=T^{2}\left(I_{\mathrm{I}}\right) I_{\mathrm{I}}
$$

is not a linear one and may have polydromic behaviour of the form indicated in figure 4 . The curve interval with a negative slope, such as the one limited by points 2 and 3, is not allowed to a physical system, that drastically switches from points 2 to $2^{\prime}$ and from 3 to $3^{\prime}$, thus behaving as a bistable device. Interval 2-3 is, then, defined by the equation

$$
\frac{\partial I_{\mathrm{T}}}{\partial I_{\mathrm{I}}}=T\left(T+2 I_{\mathrm{I}} \frac{\partial T}{\partial x^{*}} \frac{\partial x^{*}}{\partial I_{\mathrm{I}}}\right) \leqslant 0 .
$$

Obviously enough, only one term has been taken into consideration in equation (20), since the assumption has been implicitly done that experimental conditions are such as to be close to one pole position in the reduced frequency space : that pole, then, will give the maximum contribution to the factor $\frac{\partial T}{\partial I_{I}}=$ $\frac{\partial T}{\partial x^{*}} \cdot \frac{\partial x^{*}}{\partial I_{I}}$. The evaluation of the bistable behaviour of a scattering structure, then, depends on the correct evaluation of the dependence of the poles upon the impinging sound intensity. One can actually set

$$
\frac{\partial x^{*}}{\partial I_{\mathrm{I}}}=\frac{\partial x^{*}}{\partial n_{1}} \frac{\partial n_{1}}{\partial I_{\mathrm{I}}}+\frac{\partial x^{*}}{\partial n_{\mathrm{t}}} \frac{\partial n_{\mathrm{t}}}{\partial I_{\mathrm{I}}}
$$

and perform the computation of terms $\partial x^{*} / \partial n_{1}$ and $\partial x^{*} / \partial n_{\mathrm{t}}$. The width of the resonance peak will, then, be responsible for the onsetting of the bistability behaviour, together with the nonlinear strength of the scattering medium.

No quantitative analysis will be presented, here, since the qualitative aspects have. been more stressed on. In this respect, a final consideration is worth to be presented, here, and it concerns the case in which two or more poles come close together, in such a way that their effects overlap in producing an $I_{\mathrm{T}}\left(I_{\mathrm{I}}\right)$ dependence of the type represented in figure 5 . In this case, the evolution of the system, for increasing of the intensity of the impinging sound wave, takes place through the sequence of points $1-2-2^{\prime}-4-4^{\prime}$ and back through $5-5^{\prime}-3-3^{\prime}-1$. Once again, positive slope intervals $(1-2,3-4,5-6)$ are stable, while negative slope ones $(2-3,4-5)$ are forbidden to the system.

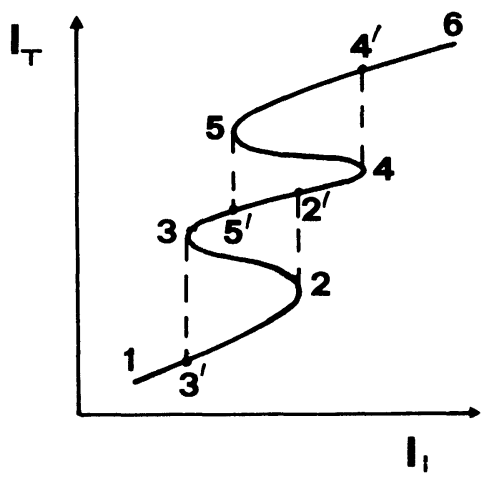

Fig. 5. - Qualitative analytical dependence of function $I_{\mathrm{T}}=I_{\mathrm{T}}\left(I_{\mathrm{I}}\right)$ in case on nonlinear scattering system : multistable behaviour produced by the partial overlapping of two resonance scattering poles.

In conclusion, a qualitative description has been given of the possibility, for a sound scattering system, to behave in a bistable way. A resonant structure is needed, where the transmission function gets a maximum value and is strongly affected by a change in the frequency or in the wavenumber. The nonlinear response of the sound velocity to a change of the sound intensity provides the necessary wavenumber variation, without changing the frequency. A final consideration has been given to the possibility of multistability insurgence, where resonant poles come close enough and add their effects in a proper way.

\section{References}

[1] McCall, S. L., Phys. Rev. A 9 (1974) 1515.

[2] Gibbs, H. M., McCall, S. L. and Venkatesan, T. N. C., in Coherence in Spectroscopy and Modern Physics, ed. by F. T. Arecchi, R. Bonifacio and M. O. Schully (Plenum, New York, N.Y.) 1978.

[3] Field, M. E. and Chen, C. L., IEEE Ultrasonics Symp. Proc. (Cherry Hill, N.J.) 1978, p. 469.

[4] Alippi, A., Bertolotti, M., Sette, D., Sibilia, C. and Shkerdin, G. N., Phys. Rev. A 30 (1984) 1883.
[5] Flax, L., Gaunaurd, G. C. and Überall, H., in Physical Acoustics XY, p. 191-294, ed. by W. P. Mason and R. N. Thurston (Academic Press, New York, N.Y.) 1981.

[6] Madigosky, W. and Fiorito, R., J. Acoust. Soc. Am. 65 (1979) 1105

[7] Fiorito, R., Madigosky, W. and Überall, H., $J$. Acoust. Soc. Am. 66 (1979) 1857.

[8] Maze, G., Ripoche, J., Durem, A. and Rousselot, J. L., Acustica 55 (1984) 27. 